\title{
Adicción al cibersexo en función de la orientación sexual: prevalencia y predictores
}

\section{Cybersex addiction according to sexual orientation: prevalence and predictors}

Fecha de recepción: 24-07-2020

Fecha de aceptación: 23-11-2020

\author{
Jesús Castro-Calvo \\ Dpto. de Personalidad, \\ Evaluación y Tratamientos Psicológicos de la Universitat de València. España. \\ Rafael Ballester-Arnal \\ Dpto. de Psicología Básica, Clínica y Psicobiología \\ Universitat Jaume I de Castellón. España. \\ Marta García-Barba \\ Dpto. de Psicología Básica, Clínica y Psicobiología, \\ Universitat Jaume I de Castellón. España.
}

\section{resumen/ahstract:}

El cibersexo, una práctica sexual generalizada en la sociedad, puede entrañar ciertos beneficios, aunque también conlleva riesgos (como desarrollar una adicción). Dado que el colectivo LGTBI (Lesbianas, gays, transexuales, bisexuales e intersexuales) lo consume más frecuentemente, el riesgo de adicción también podría ser mayor. Este estudio pretende analizar si la orientación sexual aumentaba el riesgo de consumo adictivo de cibersexo, y si la preferencia por determinadas Actividades Sexuales Online (ASOs) y las motivaciones para su realización explicaban este incremento. A tal fin, 553 personas de entre $18-40$ años $(54.2 \%$ hombres; $36.2 \%$ heterosexuales, $36.2 \%$ bisexuales, $27.7 \%$ homosexuales) completaron una encuesta online sobre consumo de cibersexo. En hombres, los homosexuales presentaban mayor severidad de consumo (pero sólo en ASOs sociales [p<.001]). En mujeres, se documentaron diferencias en función de la orientación sexual en prácticamente todos los indicadores de severidad del consumo (siendo las bisexuales las que presentaban mayor riesgo). La orientación sexual apenas influyó sobre la capacidad predictiva del tipo de ASO y sus motivaciones en el riesgo de consumo adictivo. Estos hallazgos evidencian la importancia de la orientación sexual a la hora entender el uso y abuso del cibersexo, así como la necesidad de orientar nuestras acciones preventivas hacia colectivos vulnerables.

Cybersex, a widespread sexual practice in society, can have certain benefits, but it also carries risks (such as developing an addiction). Given that the LGTBI group (Lesbians, gays, transsexuals, bisexuals and intersex) consumes it more frequently, the risk of addiction could also be higher. This study aims to analyze if sexual orientation increased the risk of addictive consumption of cybersex, and if the preference for certain Online Sexual Activities (ASOs) and the motivations fueling this behavior explained this increase. To this end, 553 people between 18-40 years old (54.2\% men; $36.2 \%$ heterosexual, $36.2 \%$ bisexual, $27.7 \%$ homosexual) completed an online survey on cybersex consumption. In men, homosexuals presented greater severity of consumption (only in social ASOs [p <.001]). In women, differences based on sexual orientation were documented in almost all the indicators of severity of consumption (with bisexuals being presenting highest risk). Sexual orientation had little influence on the predictive power of the type of ASO and its motivations over the risk of addictive consumption. These findings highlighted the relevance of sexual orientation in understanding the use and abuse of cybersex, as well as the need to target our preventive actions towards vulnerable groups.

\section{palabras clave/keywords:}

Cibersexo; adicción; orientación sexual; motivaciones.

Cybersex, addiction, sexual orientation, motives

Rafael Ballester-Arnal. Universidad Jaume I de Castelló. España Correspondencia: rballest@uji.es 


\section{Introducción}

Internet y las Tecnologías de la Información y la Comunicación (TICs) ocupan parcelas cada vez más amplias de nuestro día a día: son prácticamente indispensables para trabajar o estudiar, las usamos como forma de ocio y entretenimiento, para relacionarnos con amigos o conocer gente nueva, y también como forma de expresión de la sexualidad. El término cibersexo denomina la intersección entre la sexualidad e internet, y se definiría como «el uso de internet con objetivo de gratificación sexual» (Cooper y Griffin-Shelley, 2002). El cibersexo comprende múltiples Actividades Sexuales Online (ASOs): algunas de ellas se realizarían en solitario, mientras que otras requerirían de interacción con otros usuarios (Shaughnessy, Byers y Walsh, 2011). Las motivaciones, características, la prevalencia y el riesgo asociado a cada una de estas ASO varía, de modo que conviene diferenciarlas para comprender sus idiosincrasias (Wéry y Billieux, 2017).

Entre las ASOs que no requieren del contacto con otros usuarios, la más popular sería el consumo de pornografía. La realización de esta ASO se acompaña habitualmente de la masturbación (Daneback, Sevcikova, Månsson y Ross, 2013), de modo que la principal motivación que impulsa este comportamiento suele ser la búsqueda de la gratificación sexual inmediata. El hecho de inspirarse y aprender sobre sexo durante el visionado de pornografía también constituye otra importante motivación (Castro-Calvo, García-Barba, Gil-Juliá, Morell-Mengual y Ballester-Arnal, 2018). Hasta hace no mucho tiempo, se afirmaba que la prevalencia del consumo de pornografía dependía del género y la edad. En función del género, el consumo de pornografía era superior en los hombres (Ballester-Arnal, CastroCalvo, Gil-Llario y Giménez-García, 2014). En cuanto a su evolución durante el ciclo vital, el consumo de pornografía parecía seguir una tendencia en forma de U invertida: era poco frecuente en niños y preadolescentes, aumentaba exponencialmente entre jóvenes y adultos y volvía a decaer en personas mayores (Wéry y Billieux, 2016, 2017; Wolak, Mitchell y Finkelhor, 2007). Estudios más recientes (Ballester-Arnal, Castro-Calvo, García-Barba, Ruiz-Palomino y Gil-Llario, 2020) demuestran sin embargo que el consumo a día de hoy tanto de pornografía como de cualquier otra ASO es frecuente independientemente del género y la edad.

Entre las ASOs que sí implican interacción con otros usuarios, debemos distinguir entre aquellas que también se acompañan en ocasiones de masturbación (como mantener conversaciones sexuales con otros usuarios -a través de chat o webcam-o el intercambio de contenido sexual de producción propia a través de móvil mediante aplicaciones de mensajería instantánea o redes sociales -Sexting-) y las que no. Entre estas últimas, destacaría el uso de aplicaciones móviles basadas en geolocalización (p.e., Tinder o Grindr) para conocer a gente con la que iniciar una relación romántica y/o sexual (Rochat, Bianchi-Demicheli, Aboujaoude y Khazaal, 2019). En este último caso, la gratificación sexual no se produciría durante la realización de la ASO, sino a posteriori (cuando se contacta offline con la persona que se ha conocido). Las motivaciones para realizar este tipo de ASOs seguirían teniendo un componente de satisfacción del deseo, pero ganarían peso otras motivaciones de carácter social o afectivo (p.e., conocer gente con quien mantener relaciones románticas o sexuales online/offline, explorar preferencias sexuales alternativas, lograr intimidad emocional con 
otra persona, etc.) (Castro-Calvo, Giménez-García, Gil-Llario y Ballester-Arnal, 2018). La etapa donde el contacto sexual con otros usuarios resulta más frecuente sería entre los 2640 años, siendo similar la prevalencia en hombres y mujeres (Ballester-Arnal et al., 2020).

Existe un considerable debate en torno a los beneficios y los posibles riesgos que entrañaría el consumo de cibersexo. En la mayoría de casos, el consumo de cibersexo no supone problema alguno (Ballester-Arnal et al., 2014); al contrario, constituye una fuente de placer sexual (Daneback et al., 2013), permitiría suplir la falta de conocimiento sexual (p.e., aprendiendo nuevas prácticas sexuales) (Smith, 2013) o facilitaría encontrar parejas sexuales en un entorno seguro (Courtice y Shaughnessy, 2018), entre otros beneficios. Sin embargo, dependiendo de la forma en la que se realice, el consumo de cibersexo también podría suponer ciertos riesgos. Uno de los principales riesgos sería desarrollar un cuadro clínico de adicción al cibersexo (Duffy, Dawson y das Nair, 2016). La adicción al cibersexo se define como el «uso de cibersexo excesivo e incontrolado que desemboca en problemas laborales, sociales y personales» (Cooper y Griffin-Shelley, 2002). Según Cooper (1998), los pacientes con una adicción al cibersexo invertirían una cantidad de tiempo exagerada en la actividad sexual online, persistirían a pesar de las consecuencias negativas, no tendrían control sobre su inicio o su finalización, negarían su problema y fallarían en los intentos por controlarlo. Estos síntomas coincidirían con la propuesta del «trastorno por comportamiento sexual compulsivo», incluido en la reciente revisión de la Clasificación Internacional de Enfermedades de la OMS (CIE-11). De acuerdo con Kafka (2013), otro síntoma sería el uso del cibersexo como regulador emocional (principalmente de estados de ansiedad/ depresión). En cuanto a su prevalencia, en nuestro país el porcentaje de adolescentes que abusaba del cibersexo se situaba entre el $8.6 \%$ (chicos) y $2.2 \%$ (chicas) (Ballester-Arnal, Giménez-García, Gil-Llario y Castro-Calvo, 2016b); en adultos, el 90\% mostraba un perfil de bajo riesgo de adicción, el $8.6 \%$ de riesgo medio y el $0.7 \%$ de alto riesgo (BallesterArnal, Castro-Calvo, Gil-Llario y Gil-Julià, 2016a).

\section{Consumo adictivo de cibersexo en función de la orientación sexual}

Un hallazgo sistemático en la mayoría de estudios de cibersexo es que las personas homosexuales y bisexuales lo consumirían con más frecuencia (Træen y Daneback, 2013). Y lo que sería más preocupante: parece que también lo harían de forma más problemática, siendo este riesgo 1.5 veces mayor que en la población heterosexual (Bőthe, Vaillancourt-Morel, Bergeron y Demetrovics, 2019; Ballester-Arnal, Gil-Llario, Giménez-García, Castro-Calvo y Cárdenas-López, 2017). Este aumento en el consumo de cibersexo y el riesgo asociado se aprecia en todas las ASOs, pero en especial en aquellas que implican contacto con otros usuarios: así, las personas con una orientación alternativa a la heterosexual utilizarían en mucha mayor medida internet para conocer personas con las que mantener relaciones románticas/sexuales (Seal et al., 2015), lo que también podría aumentar su vulnerabilidad a la hora de desarrollar una adicción. Para explicar esta popularidad, algunos recurren a la metáfora del «oasis erótico» (Ross, Tikkanen y Mansson, 2000, p. 750): en contextos en los que no ser heterosexual supone una cierta sanción social, internet permitiría que una persona homosexual o bisexual acceda a contenidos y actividades sexuales ajustados a sus preferencias y que además lo haga de forma anónima. Estas personas encontrarían en Internet 
-y en el anonimato que garantiza- el entorno ideal donde explorar su sexualidad sin temor al estigma. Estas hipótesis se ajustarían bien a la teoría del uso compensatorio de internet (Kardefelt-Winther, 2014), que propone que el uso recreativo y sobre todo el consumo excesivo de cualquier tipo de actividad online (en este caso, de la ASO) se puede explicar a partir de las necesidades que permite compensar.

A pesar de la solidez de los hallazgos que demuestran que la orientación sexual condiciona varias dimensiones del consumo de cibersexo, apenas disponemos de estudios que analicen si esto supone un riesgo mayor de consumo adictivo. Así, el objetivo de este estudio fue analizar si la orientación sexual aumenta la gravedad de consumo de cibersexo (medido mediante varios indicadores diagnósticos). Asimismo, recientemente realizamos un estudio en el que demostrábamos que las preferencias por distintos tipos de ASO y las motivaciones tras estos comportamientos variaban en función de la orientación sexual (Castro-Calvo, GilLlario, Giménez-García, Gil-Juliá y Ballester-Arnal, 2020). Sabemos que estos aspectos también condicionan el riesgo de consumo adictivo, de modo que un segundo objetivo fue explorar si las preferencias por determinadas ASOs y la presencia de ciertas motivaciones detrás de este consumo podrían explicar el riesgo diferencial de adicción al cibersexo en función de la orientación sexual.

\section{Método}

\section{Procedimiento y participantes}

Los participantes en esta investigación fueron reclutados y evaluados a través de ADISEX (http://adiccionalsexo.uji.es/), una plataforma que constituye un referente en habla hispana diseñada para la evaluación y el tratamiento de los problemas en el control de los impulsos sexuales. Esta plataforma permite que cualquier persona acceda gratuitamente a una completa evaluación del control de sus impulsos sexuales online y offline, así como a tratamiento en el caso de que sus resultados sugieran la presencia de problemas en este ámbito. La página es accesible a través de cualquier motor de búsqueda usando términos como: "adicción al cibersexo", "adicciones sexuales", "evaluación de la adicción al cibersexo" o "tratamiento de la adicción al cibersexo". Esta estrategia de reclutamiento se complementó con otras que permitieran enriquecer el perfil y número de participantes en el estudio. A tal fin, empleamos el sistema de publicidad de Facebook (publicaciones sugeridas) para lanzar campañas de difusión en las que se solicitaba colaboración para un estudio sobre sexualidad.

A través de estos procedimientos, se reclutó a una muestra representativa de 8,040 hombres y mujeres provenientes de todo el territorio español. A fin de ajustar la muestra al objetivo del estudio, para este artículo se seleccionó a una submuestra de 553 personas de entre 1840 años $(M=24.73 ; D T=5.39)$. La selección de esta submuestra no fue azarosa, sino que se realizó a fin de que resultara equitativa en términos de distribución por género y orientación sexual. Así, el 54.2\% de los participantes eran hombres, y el 45.8\% restante mujeres. En cuanto a la orientación sexual, el $36.2 \%$ se definían heterosexuales, el 36.2\% bisexuales y el $27.7 \%$ restante homosexuales. Los 553 participantes se clasificaron en seis grupos a partir del cruce entre las variables anteriores: hombres heterosexuales $(n=100)$, bisexuales $(n=100)$ y gays $(n=100)$, mujeres heterosexuales $(n=100)$, bisexuales $(n=100)$ y lesbianas 
$(n=53)$. En la Tabla 1 se detallan y comparan algunas de sus características sociodemográficas y sexuales.

Tabla 1.- Características sociodemográficas de la muestra en función del género y la orientación sexual

\begin{tabular}{|c|c|c|c|c|c|c|c|c|}
\hline & \multicolumn{4}{|c|}{ Hombres (n=300) } & \multicolumn{4}{|c|}{ Mujeres (n=253) } \\
\hline & $\begin{array}{c}\text { Heterosexuales } \\
\text { M(DT) } 0 \%\end{array}$ & $\begin{array}{l}\text { Bisexuales } \\
\text { M(DT) } 0 \%\end{array}$ & $\begin{array}{l}\text { Homosexuales } \\
\text { M (DT) } 0 \%\end{array}$ & $\begin{array}{l}\text { Estadístico } \\
\text { diferencial }\end{array}$ & $\begin{array}{l}\text { Heterosexuales } \\
\text { M (DT) } 0 \%\end{array}$ & $\begin{array}{c}\text { Bisexuales } \\
\text { M(DT) } \\
0 \%\end{array}$ & $\begin{array}{l}\text { Homosexuales } \\
\text { M (DT) } 0 \%\end{array}$ & $\begin{array}{l}\text { Estadístico } \\
\text { diferencial }\end{array}$ \\
\hline Edad media $(D T)$ & $27.2(6.0)$ & $25.4(5.8)$ & $25.8(5.6)$ & $F=2.51$ & $23.1(4.1)$ & $22.4(4.1)$ & $23.7(4.1)$ & $F=1.76$ \\
\hline \multicolumn{9}{|l|}{ Tipo de relación } \\
\hline Sin pareja & $28 \%$ & $44 \%$ & $49 \%$ & & $28 \%$ & $24 \%$ & $37.7 \%$ & \\
\hline Pareja estable & $60 \%$ & $40 \%$ & $37 \%$ & $X^{2}=13.38^{* *}$ & $55 \%$ & $54 \%$ & $54.7 \%$ & $X^{2}=6.57$ \\
\hline Parejas esporádicas & $12 \%$ & $16 \%$ & $14 \%$ & & $17 \%$ & $22 \%$ & $7.5 \%$ & \\
\hline Ordenador propio (sí) & $97 \%$ & $97 \%$ & $100 \%$ & $X^{2}=3.06$ & $98 \%$ & $97 \%$ & $100 \%$ & $X^{2}=1.61$ \\
\hline $\begin{array}{l}\text { Tiempo semanal medio } \\
\text { online en horas }(D T)\end{array}$ & $31.1(26.8)$ & $26.1(26.6)$ & $28.3(22.7)$ & $F=0.96$ & $27.3(21.6)$ & $35.7(27.2)$ & $20.8(15.8)$ & $F=7.85^{* * *}$ \\
\hline \multicolumn{9}{|l|}{$\begin{array}{l}\text { Comportamiento } \\
\text { sexual }\end{array}$} \\
\hline $\begin{array}{l}\text { Relaciones con } \\
\text { personas del otro } \\
\text { sexo (sí) }\end{array}$ & $88 \%$ & $79 \%$ & $24 \%$ & $X^{2}=103.78^{* * *}$ & $90 \%$ & $88 \%$ & $58.5 \%$ & $X^{2}=57.18 * * *$ \\
\hline $\begin{array}{l}\text { Relaciones con } \\
\text { personas del mismo } \\
\text { sexo (sí) }\end{array}$ & $13 \%$ & $75 \%$ & $97 \%$ & $X^{2}=160.52^{* * *}$ & $10 \%$ & $54 \%$ & $88.7 \%$ & $X^{2}=93.96 * * *$ \\
\hline Masturbación(sí) & $99 \%$ & $98 \%$ & $100 \%$ & $X^{2}=2.01$ & $94 \%$ & $100 \%$ & $90.6 \%$ & $X^{2}=8.49^{*}$ \\
\hline Sexo oral (sí) & $84 \%$ & $89 \%$ & $93 \%$ & $X^{2}=4.04$ & $87 \%$ & $94 \%$ & $84.9 \%$ & $X^{2}=3.94$ \\
\hline Sexo vaginal (sí) & $84 \%$ & $74 \%$ & $19 \%$ & $X^{2}=101.28^{* * *}$ & $91 \%$ & $88 \%$ & $79.2 \%$ & $X^{2}=4.39$ \\
\hline Sexo anal (sí) & $51 \%$ & $66 \%$ & $93 \%$ & $X^{2}=43.14^{* * *}$ & $44 \%$ & $48 \%$ & $17 \%$ & $X^{2}=15.04 * * *$ \\
\hline $\begin{array}{l}\text { Frecuencia sexual } \\
\text { media }(D T) \text { (rango } \\
\text { entre } 0-7 \text { ) }\end{array}$ & $5.87(0.99)$ & $5.64(1.36)$ & $5.91(0.91)$ & $F=0.93$ & $4.93(1.28)$ & $5.21(1.40)$ & $5.41(0.83)$ & $F=2.42$ \\
\hline
\end{tabular}

Nota: $*<.05 ; * * \mathrm{p}<.01 ; * * * \mathrm{p}<.001$

\section{Instrumentos}

En esta investigación, se aplicó una batería de instrumentos de evaluación que exploraban múltiples áreas del comportamiento sexual online y offline. El tiempo medio para completar todos los cuestionarios fue de 28.6 minutos $(D T=42.6)$. En este trabajo, se analizaron únicamente las siguientes variables: 


\section{Características sociodemográficas y sexuales}

Los participantes completaron un cuestionario ad hoc en el que se les preguntaba acerca de su género, edad, tipo de relación ("sin pareja", "pareja estable" o "parejas esporádicas"), orientación sexual, si disponían de ordenador con acceso a Internet y el tiempo semanal medio que pasaban online (en horas).

En cuanto a su comportamiento sexual, se preguntó a los participantes si habían mantenido relaciones sexuales con personas del otro sexo (sílno), de su mismo sexo (sílno), si habían realizado una serie de comportamientos sexuales (masturbación, sexo oral, vaginal y anal [sí/no]) y su frecuencia sexual (escala Likert con seis opciones de respuesta entre 0 ["menos de 6 veces al año"] y 7 ["más de tres veces a la semana"]).

\section{Comportamiento sexual online}

Para la evaluación de distintos aspectos del comportamiento sexual online, los participantes completaron el Cuestionario de Prácticas Sexuales Online (CPSO, Salusex, 2016). El CPSO se compone de múltiples preguntas distribuidas en diversos bloques. El formato de respuesta varía en función de la dimensión analizada, si bien en la mayoría de los casos las preguntas son de respuesta dicotómica (sílno). En primer lugar, el CPSO explora dos aspectos básicos del comportamiento sexual online: (a) si alguna vez se ha utilizado internet con objetivos sexuales (sí/no) y (b) el tiempo medio dedicado a la ASO (“ ¿cuántas horas semanales pasas conectado a páginas sexuales?"). Luego, se pregunta a los participantes acerca de la realización (sílno) de 12 tipos distintos de ASO (p.e., "visionado de imágenes o películas pornográficas", "búsqueda de pareja sexual" o "chatear reproduciendo fantasías sexuales con otro usuario"). El CPSO finaliza con un bloque de 8 ítems dicotómicos (síl no) acerca de los motivos tras del consumo de cibersexo (p.e., "encontrar material sexual con el que masturbarme" o "relajarme del estrés de mis obligaciones"). Todas las ASO y los motivos evaluados a través del CPSO se detallan en la tabla 3. La fiabilidad $(\alpha)$ de las escalas de ASO y motivaciones fue de.76 y .63. Debido a la pobre fiabilidad de los ítems tomados en conjunto (es decir, como factores), en esta investigación los usamos a modo de indicadores independientes.

\section{Severidad del consumo de cibersexo}

La severidad del consumo de cibersexo se evaluó usando la adaptación española del Cuestionario de Adicción al Cibersexo (ISST, Ballester-Arnal, Gil-Llario, Gómez-Martínez y Gil-Julià, 2010). El ISST, desarrollado originalmente por Delmonico (1997), se compone de 25 preguntas con formato de respuesta dicotómica (verdadero/falso) que evalúan el grado en que la conducta sexual online resulta o no problemática. La suma de sus ítems permite obtener un índice general de adicción al cibersexo, así como puntuaciones en cinco factores: (a) compulsividad sexual online (pérdida del control sobre la ASO [p.e., "cuando no consigo acceder a información sexual online me siento ansioso, enfadado o decepcionado"]); (b) cibersexo solitario (implicación en ASO que no requieren interactuar con otros usuarios online [p.e., "he buscado material sexual a través de un buscador de Internet"]); (c) cibersexo social (realización de ASO que sí requieren interactuar con otros usuarios online [p.e., "he participado en chats sexuales"]); (d) gasto económico online (inversión de dinero durante 
la ASO [p.e., "me he afiliado a sitios sexuales para conseguir acceder a material sexual online"]); y (e) percepción de severidad del comportamiento sexual online (consideración de que la ASO puede estar resultando problemática [p.e., "me he castigado a mí mismo cuando uso Internet con fines sexuales"]). En la validación española, Ballester-Arnal et al. (2010) encontraron una fiabilidad de .88 para la escala general, y de entre .49-.81 para las subescalas. En el presente estudio, la fiabilidad de la escala global fue de .85 , y de entre .54 y .74 para los factores.

Algunos investigadores han propuesto puntos de corte que permiten usar el ISST para clasificar a los usuarios de cibersexo en tres perfiles (Carnes, Delmonico y Griffin, 2001): usuarios recreativos (puntuaciones entre 0-8), de riesgo (entre 9-18) y adictos (entre 19-25). Si bien no existen estudios que exploren la sensibilidad y especificidad diagnóstica de estos criterios, se han empleado en diversas investigaciones para identificar la presencia de posible consumo patológico (Ballester-Arnal et al., 2016b; Castro-Calvo et al., 2020).

\section{Percepción de la gravedad del consumo de cibersexo}

Finalmente, se plantearon cuatro preguntas de respuesta dicotómica (sí/no) a fin de evaluar la percepción de gravedad del consumo de cibersexo en distintos ámbitos: (a) preocupación derivada de la ASO (“¿alguna vez te ha preocupado tu consumo de cibersexo?”); (b) percepción de dedicación excesiva ("¿alguna vez has pensado que le dedicabas demasiado tiempo?”); (c) percepción de interferencia (“¿el cibersexo ha interferido con aspectos importantes de tu vida?"); y (d) percepción de adicción (“' te consideras adicto al cibersexo?").

\section{Análisis de datos}

Los datos se analizaron mediante el paquete estadístico SPSS Versión 25.0. En primer lugar, se realizaron análisis descriptivos para caracterizar a los participantes en términos sociodemográficos y de comportamiento sexual, analizando la existencia de diferencias significativas en función de la orientación sexual. Las diferencias en función de la orientación se evaluaron a través de pruebas ANOVA de un factor (variables continuas) y contrastes Chi Cuadrado (variables categóricas). Esta misma aproximación metodológica se empleó posteriormente para analizar diversos indicadores de consumo problemático de cibersexo en función de la orientación sexual. Finalmente, se realizaron diversas regresiones lineales (una por cada uno de los 6 grupos en los que se clasificó a la muestra) a través del método por pasos sucesivos; el objetivo fue analizar la capacidad predictiva de distintos tipos de ASO y motivaciones (variables independientes) sobre la severidad del consumo de cibersexo (variable dependiente). La adecuación de los modelos de regresión se analizó a partir de su ajuste (significación de $F$ ) y de su capacidad explicativa sobre la varianza de la severidad del consumo $\left(R^{2}\right)$. A fin de que los resultados de los distintos modelos resultaran comparables, la capacidad predictiva de las variables se presenta usando valores estandarizados ( $\beta$ estandarizada).

Para evitar que las diferencias en el consumo de cibersexo de hombres y mujeres dificultaran el análisis de las diferencias en función de la orientación sexual (es decir, el objetivo del estudio), los análisis se realizaron para cada sexo por separado (Ballester-Arnal et al., 2020). 


\section{Resultados}

Consumo problemático de cibersexo en función de la orientación sexual

En la Tabla 2, se analizan distintos indicadores de consumo problemático de cibersexo en función de la orientación sexual. En hombres, no se aprecian diferencias significativas en el tiempo medio dedicado a la ASO en función de la orientación sexual $(F=0.82)$, pero sî en cuanto a la gravedad del consumo de cibersexo. En concreto, los participantes homosexuales obtuvieron mayor puntuación en el uso social del cibersexo $(F=34.27 ; \mathrm{p}<.001)$; sin embargo, su percepción de gravedad (medido a través del ISST) resultó significativamente menor $(F=16.77)$, sobre todo cuando se compara con el obtenido por los heterosexuales $(M=0.71$ vs. $M=1.52)$. En línea con estos resultados, los heterosexuales también respondieron afirmativamente con mayor frecuencia a 3 de las 4 preguntas sobre percepción de la gravedad del consumo de cibersexo: preocupación derivada de la ASO (59.6\% en heterosexuales frente al $40.8 \%$ y el $49.5 \%$ en bisexuales y homosexuales), percepción de dedicación excesiva (55.7\% frente al $32.3 \%$ y el $31.6 \%$ ) y percepción de adicción (51\% frente al $27 \%$ y $28 \%$ ). Finalmente, no encontramos diferencias en la prevalencia de adicción al cibersexo en función de la orientación sexual, si bien la proporción en bisexuales y homosexuales (8\%) doblaba a la encontrada en heterosexuales (4\%).

En mujeres, encontramos diferencias significativas en función de la orientación sexual en muchos de los indicadores de consumo problemático analizados, en su mayoría indicando una mayor puntuación entre las bisexuales. En cuanto al tiempo online con fines sexuales, las mujeres bisexuales invertían una media semanal de 98.08 minutos (frente a 64.2 y 54.7 en heterosexuales y homosexuales; $F=3.24$ ). Las mujeres bisexuales también obtuvieron una puntación mayor en el índice general de severidad del consumo del ISST $(F=7.65)$, así como en los factores de compulsividad sexual online $(F=5.01)$, cibersexo solitario $(F=10.01)$ y cibersexo social $(F=4.81)$. Sin embargo, no se aprecian diferencias en el porcentaje de respuestas positivas a ninguno de los ítems de percepción de gravedad del consumo de cibersexo. En cuanto a la prevalencia de los distintos perfiles de consumo de cibersexo, nuevamente aparecen diferencias significativas en función de la orientación $\left(X^{2}=16.99\right)$. En este caso, la proporción de mujeres bisexuales con un consumo de riesgo superaba ampliamente la observada en heterosexuales y homosexuales (33\% frente al $12 \%$ y el $13 \%$ respectivamente); sin embargo, ninguna mujer bisexual se calificó como adicta al cibersexo, pero sí un $1 \%$ de las heterosexuales y un $2.2 \%$ de las homosexuales.

\section{Predictores del riesgo de adicción al cibersexo en función de la orientación sexual}

En la Tabla 3 se incluyen los resultados de las regresiones lineales que analizan el riesgo de adicción al cibersexo (puntuación total en el ISST) a partir de distintos tipos de ASO y las motivaciones para su realización. Como se aprecia, todos los modelos fueron significativos $(\mathrm{p}<.001)$ y con capacidades predictivas que oscilaban entre el $38.3 \%-44.1 \%$ en hombres y el 52.1\%-58.6\% en mujeres. Si bien ya apuntábamos la inconveniencia de realizar comparaciones en función del género en este caso sí resultaría interesante analizar los predictores en función de esta variable. Destacaría el hecho de que mientras en los hombres, los tipos de ASOs y sus motivaciones tienen una capacidad predictiva semejante ( $\beta$ entre .18-.32 y .18- 
Tabla 2.- Indicadores de consumo problemático de cibersexo en función de la orientación sexual

\begin{tabular}{|c|c|c|c|c|c|c|c|c|}
\hline & \multicolumn{4}{|c|}{ Hombres ( $\mathrm{n}=300$ ) } & \multicolumn{4}{|c|}{ Mujeres (n=253) } \\
\hline & $\begin{array}{l}\text { Heterosexuales } \\
\text { M (DT) } 0 \%\end{array}$ & $\begin{array}{l}\text { Bisexuales } \\
\text { M (DT) } 0 \%\end{array}$ & $\begin{array}{l}\text { Homosexuales } \\
\text { M (DT) } 0 \%\end{array}$ & $\begin{array}{l}\text { Estadistico } \\
\text { diferencial }\end{array}$ & $\begin{array}{l}\text { Heterosexuales } \\
\text { M(DT) } 0 \%\end{array}$ & $\begin{array}{l}\text { Bisexuales } \\
\text { M (DT) } 0 \%\end{array}$ & $\begin{array}{l}\text { Homosexuales } \\
\text { M(DT) } 0 \%\end{array}$ & $\begin{array}{l}\text { Estadístico } \\
\text { diferencial }\end{array}$ \\
\hline $\begin{array}{l}\text { Tiempo semanal medio en } \\
\text { minutos dedicado a la ASO } \\
(D T)\end{array}$ & $259.1(255.5)$ & $216.53(207.1)$ & $241.3(227.7)$ & $F=0.82$ & $64.2(111.27)$ & $98.08(133.2)$ & $54.7(58.6)$ & $F=3.24^{*}$ \\
\hline \multicolumn{9}{|l|}{$\begin{array}{l}\text { Severidad del consumo } \\
\text { de cibersexo (Internet Sex } \\
\text { Screening Test) }\end{array}$} \\
\hline $\begin{array}{l}\text { Puntuación total (rango } \\
\text { entre 0-25) }\end{array}$ & $10.83(4.29)$ & $11.32(4.90)$ & $11.54(453)$ & $F=0.62$ & $5.37(3.64)$ & $7.16(3.29)$ & $5.34(3.79)$ & $F=7.65^{* * *}$ \\
\hline $\begin{array}{l}\text { Compulsividad sexual } \\
\text { online (rango entre 0-8) }\end{array}$ & $2.62(1.91)$ & $2.38(1.96)$ & $2.24(1.93)$ & $F=0.98$ & $0.63(1.14)$ & $1.06(1.17)$ & $0.52(1.06)$ & $F=5.01 * *$ \\
\hline $\begin{array}{l}\text { Cibersexo solitario (rango } \\
\text { entre } 0-6 \text { ) }\end{array}$ & $4.51(1.17)$ & $4.26(1.23)$ & $4.35(1.29)$ & $F=1.05$ & $2.79(1.51)$ & $3.62(1.28)$ & $2.84(1.38)$ & $F=10.01^{* * *}$ \\
\hline $\begin{array}{l}\text { Cibersexo social (rango } \\
\text { entre 0-6) }\end{array}$ & $1.91(1.71)$ & $3.08(1.73)$ & $3.84(1.51)$ & $F=34.27^{* * *}$ & $1.07(1.28)$ & $1.66(1.60)$ & $1.10(1.35)$ & $F=4.81^{* *}$ \\
\hline $\begin{array}{l}\text { Gasto económico online } \\
\text { (rango entre 0-2) }\end{array}$ & $0.27(0.46)$ & $0.38(0.63)$ & $0.40(0.60)$ & $F=1.49$ & $0.12(0.32)$ & $0.14(0.42)$ & $0.13(0.34)$ & $F=0.07$ \\
\hline $\begin{array}{l}\text { Percepción de severidad } \\
\text { (rango entre 0-3) }\end{array}$ & $1.52(0.93)$ & $1.22(1.13)$ & $0.71(0.85)$ & $F=16.77^{* * *}$ & $0.76(0.79)$ & $0.68(0.69)$ & $0.73(0.88)$ & $F=0.28$ \\
\hline
\end{tabular}

Percepción de gravedad del consumo de cibersexo

¿Alguna vez te ha preocupado tu consumo de cibersexo? (sí)

¿Alguna vez has pensado que le dedicabas demasiado tiempo? (sí)

¿El cibersexo ha interferido con aspectos importantes de tu vida?

$49.5 \%$

$X^{2}=6.96^{*}$

$45.7 \%$

$41.8 \%$

$47.9 \%$

$X^{2}=0.56$

$55.7 \%$

$32.3 \%$

$31.6 \%$

$X^{2}=16.23^{* * *}$

$4.3 \%$

$8.2 \%$

$2.1 \%$

$X^{2}=2.69$

(sí)

¿Te consideras adicto al

cibersexo? (sí)

$51 \%$

$27 \%$

$28 \% \quad X^{2}=16.13^{* * *}$

$3 \%$

$5 \%$

$0 \%$

$X^{2}=2.53$

Prevalencia de diferentes perfiles de consumo de cibersexo

\begin{tabular}{|c|c|c|c|c|c|c|c|c|}
\hline Consumo recreativo & $33 \%$ & $33 \%$ & $24 \%$ & & $87 \%$ & $67 \%$ & $84.8 \%$ & \\
\hline Consumo de riesgo & $63 \%$ & $59 \%$ & $68 \%$ & $X^{2}=4.04$ & $12 \%$ & $33 \%$ & $13 \%$ & $X^{2}=16.99^{* *}$ \\
\hline Adicción al cibersexo & $4 \%$ & $8 \%$ & $8 \%$ & & $1 \%$ & $0 \%$ & $2.2 \%$ & \\
\hline
\end{tabular}

Nota: ${ }^{*}<.05 ; * * \mathrm{p}<.01 ; * * * \mathrm{p}<.001$ 
Tabla 3.- Predictores del riesgo de adicción al cibersexo en función del género y la orientación sexual

\begin{tabular}{|c|c|c|c|c|c|c|}
\hline & \multicolumn{3}{|c|}{ Hombres $(n=300)$} & \multicolumn{3}{|c|}{ Mujeres (n=253) } \\
\hline & $\begin{array}{c}\text { Hetero- } \\
\text { sexuales } \\
\beta\end{array}$ & $\begin{array}{c}\text { Bisexuales } \\
\beta\end{array}$ & $\begin{array}{c}\text { Homosexuales } \\
\beta\end{array}$ & $\begin{array}{c}\text { Hetero- } \\
\text { sexuales } \\
\beta\end{array}$ & $\begin{array}{c}\text { Bisexuales } \\
\beta\end{array}$ & $\begin{array}{c}\text { Homosexuales } \\
\beta\end{array}$ \\
\hline \multicolumn{7}{|l|}{ Tipos de actividad sexual online } \\
\hline $\begin{array}{l}\text { Visionado de imágenes o películas } \\
\text { pornográficas }\end{array}$ & NS & NS & NS & $0.22 * *$ & $0.18^{* *}$ & NS \\
\hline $\begin{array}{l}\text { Búsqueda de información sobre educación } \\
\text { sexual }\end{array}$ & NS & NS & NS & NS & NS & NS \\
\hline Lectura de textos eróticos & NS & NS & $0.19^{*}$ & $0.15^{*}$ & $0.14^{*}$ & $0.38^{* *}$ \\
\hline Búsqueda en páginas Web de contactos & $0.30^{* * *}$ & NS & NS & NS & NS & NS \\
\hline Búsqueda de pareja sexual & NS & $0.18^{*}$ & $0.32 * * *$ & $0.31^{* * *}$ & $0.16^{*}$ & NS \\
\hline $\begin{array}{l}\text { Insinuaciones sexuales con conocidos } 0 \\
\text { desconocidos }\end{array}$ & NS & NS & NS & $0.27^{* * *}$ & $0.20^{*}$ & NS \\
\hline $\begin{array}{l}\text { Chatear reproduciendo fantasías sexuales } \\
\text { con otro usuario }\end{array}$ & $0.25^{* *}$ & $0.21^{*}$ & NS & NS & $0.23^{* *}$ & $0.28^{*}$ \\
\hline Búsqueda de pareja romántica & NS & NS & NS & NS & NS & NS \\
\hline $\begin{array}{l}\text { Contacto sexual por medio de Webcam } \\
\text { con otro usuario }\end{array}$ & NS & NS & NS & NS & NS & $0.29 *$ \\
\hline Respuesta a anuncios sexuales & $0.24 * *$ & NS & NS & NS & NS & NS \\
\hline Compra de material sexual online & NS & NS & NS & NS & $0.18^{* *}$ & $0.25^{*}$ \\
\hline Contacto con trabajadores/as sexuales & NS & $0.24^{* *}$ & NS & $0.33^{* * *}$ & $0.23 * * *$ & NS \\
\hline \multicolumn{7}{|l|}{ Motivaciones para la práctica del cibersexo } \\
\hline Distraerme & NS & NS & NS & NS & NS & NS \\
\hline $\begin{array}{l}\text { Encontrar material sexual con el que } \\
\text { masturbarme }\end{array}$ & NS & NS & NS & NS & NS & NS \\
\hline $\begin{array}{l}\text { Disfrutar actividades sexuales que no hago } \\
\text { habitualmente }\end{array}$ & $0.22^{* *}$ & $0.28 * * *$ & $0.18^{*}$ & NS & $0.20^{* *}$ & NS \\
\hline Aprender cosas sobre sexo & NS & NS & NS & NS & NS & NS \\
\hline Relajarme del estrés de mis obligaciones & NS & NS & NS & NS & NS & NS \\
\hline Animarme cuando estoy decaído & $0.24 *$ & NS & $0.27^{* * *}$ & NS & NS & NS \\
\hline Conocer a otras personas & NS & NS & $0.46^{* * *}$ & NS & NS & NS \\
\hline Intentar establecer relaciones & NS & $0.20^{*}$ & $0.29^{*}$ & NS & NS & NS \\
\hline \multicolumn{7}{|l|}{ Ajustes del modelo de regresión lineal (por pasos) } \\
\hline$F$ & $14.82^{* * *}$ & $12.68 * * *$ & $9.60^{* * *}$ & $24.60^{* * *}$ & $16.11^{* * *}$ & $11.12^{* * *}$ \\
\hline$R^{2}$ & $44.1 \%$ & $40.3 \%$ & $38.3 \%$ & $56.7 \%$ & $58.6 \%$ & $52.1 \%$ \\
\hline
\end{tabular}

Nota: $\mathrm{NS}=$ No significativo; ${ }^{*}<.05 ; * * \mathrm{p}<.01 ; * * * \mathrm{p}<.001$ 
.46 respectivamente), en las mujeres lo que determinaría el riesgo de adicción al cibersexo serían principalmente los tipos de ASOs y no tanto las motivaciones. Así, sólo una motivación ("disfrutar de actividades sexuales que no hago habitualmente") mostró capacidad predictiva sobre el riesgo de adicción al cibersexo, mientras que un abanico de ASOs sí resultaron predictores eficaces de esta variable dependiente ( $\beta$ entre .14-.38).

El segundo nivel de análisis pasaría por comparar los predictores del riesgo de adicción al cibersexo en función de la orientación sexual (en este caso, distinguiendo ya en función del género). En este sentido, parece que los resultados indican que son pocos los indicadores de riesgo diferenciales en función de la orientación; o, dicho de otro modo, parece que lo que aumenta el riesgo en heterosexuales lo haría también en homosexuales y bisexuales. Si analizamos con un poco más de detalle el patrón de valores predictivos, encontramos algunas excepciones a esta afirmación. En hombres, encontramos dos ASO con capacidad predictiva sobre la severidad del consumo únicamente en heterosexuales: la búsqueda en web de contactos $(\beta=0.30)$ y la respuesta a anuncios sexuales $(\beta=0.24)$. En hombres bisexuales, el hecho de contactar con trabajadores sexuales resultó ser un predictor positivo de la severidad del consumo $(\beta=0.24)$. Finalmente, una ASO (la lectura de textos eróticos; $\beta=0.19$ ) y una motivación (usar cibersexo para conocer otras personas; $\beta=0.46$ ) eran predictores únicos del riesgo de consumo problemático en homosexuales. En mujeres, ninguna de las variables exploradas resultaron indicadores únicos del riesgo en heterosexuales. En el caso de las bisexuales, lo fue una motivación ("disfrutar de actividades sexuales que no hago habitualmente"; $\beta=0.20$ ) y en el de las homosexuales, una ASO ("mantener contacto sexual a través de la cámara web"; $\beta=0.29$ ).

\section{Discusión y conclusiones}

El objetivo del presente estudio era analizar si la orientación sexual aumentaba el riesgo de consumo adictivo de cibersexo, y si la preferencia por determinadas ASOs y la presencia de ciertas motivaciones podrían explicar este riesgo.

Según indican nuestros resultados, en los hombres, apenas se aprecian diferencias significativas en la severidad del consumo en función de la orientación sexual. La única excepción la constituiría el uso social del cibersexo, donde la puntuación es mayor entre los hombres homosexuales. Este incremento podría atribuirse al hecho de que muchos hombres homosexuales encuentren en internet un medio seguro que les permita encontrar parejas (tanto sexuales como románticas), lo que haría que este aspecto (que, en principio, sería beneficioso) resulte problemático (Courtice y Shaughnessy, 2018). No obstante, en nuestro estudio eran los hombres heterosexuales los que reportaban mayor percepción de gravedad. Estos resultados paradójicos pueden deberse a que la interferencia derivada del consumo de cibersexo (es decir, la percepción de síntomas negativos) sea mayor entre los hombres heterosexuales que entre los homosexuales, explicando que aun experimentando un riesgo objetivo mayor, los homosexuales valoren más indulgentemente su ASO.

En el caso de las mujeres, sí encontramos diferencias significativas en función de la orientación sexual en cuanto a la severidad del consumo de cibersexo. Concretamente, las mujeres bisexuales presentan mayor riesgo de consumo problemático de cibersexo en prácticamente 
todos los indicadores analizados. Al igual que en los hombres, las mujeres con una orientación sexual diferente a la heterosexual también encontrarían en internet un espacio seguro donde experimentar con su sexualidad, por lo que también pueden ser más propensas a utilizarlo con una mayor frecuencia y hacerlo de forma más problemática (Daneback, Cooper y Mansson, 2005). En el caso de las mujeres bisexuales, las ASOs además podrían resultar importantes a la hora de completar las preferencias sexuales insatisfechas durante sus relaciones offline (p.e., una chica bisexual con una pareja masculina offline que usaría internet para buscar pornografía lésbica). Nuevamente, las diferencias objetivas en cuanto a severidad del consumo de cibersexo no concordaban con un incremento en la percepción de su gravedad, posiblemente porque las ventajas que suponía para las mujeres bisexuales su consumo de cibersexo (p.e., conocer parejas sexuales) tenían más peso que las posibles consecuencias negativas derivadas de este consumo.

Otro de los objetivos era comprobar si el tipo de ASOs y las motivaciones pueden explicar el riesgo de adicción al cibersexo en función de la orientación sexual y el género. En hombres, tanto los tipos de ASO como las motivaciones resultaron ser potencialmente predictoras de la severidad del consumo de cibersexo; sin embargo, en mujeres solo lo fueron las ASOs. En otras palabras: parece que, en mujeres, el riesgo de consumo adictivo no dependería tanto de porqué se consume cibersexo como del tipo de ASO que se realice. En cuanto a la orientación sexual, apenas se encontraban marcadores de riesgo diferencial en función de esta variable. Por ejemplo, la mayoría de ASOs que requerían participación de otros usuarios fueron predictoras de la severidad del consumo en las tres orientaciones sexuales, y tan sólo algunas ASOs aisladas (como la lectura de textos eróticos en hombres homosexuales o la respuesta a anuncios sexuales en heterosexuales) fueron predictoras en una orientación sexual concreta. Algo muy similar sucedería en el caso de las mujeres, donde apenas se apreciaría algún marcador de riesgo diferencial aislado en función de la orientación sexual (p.e., el uso de webcams con fines sexuales en mujeres homosexuales). Es decir, que las ASOs y las motivaciones mostrarían una capacidad predictiva semejante en cualquier orientación sexual.

En definitiva, como hemos podido observar a lo largo de estas líneas, parece ser que las personas con una orientación sexual diferente a la heterosexual difieren de los y las heterosexuales en cuanto a la severidad del consumo de cibersexo y la percepción de gravedad. Sin embargo, las preferencias por determinadas ASOs y la presencia de ciertas motivaciones detrás del consumo de cibersexo apenas explican este riesgo diferencial en función de la orientación sexual, por lo que habría que explorar con mayor profundidad los predictores de este riesgo diferencial. Estos datos evidencian la necesidad de trabajar de forma diferenciada, en función de la orientación sexual, la percepción de la gravedad y de las consecuencias negativas del uso del cibersexo para lograr una detección precoz de los casos en los que haya un consumo problemático.

\section{Limitaciones y direcciones futuras}

Una de las principales limitaciones de este estudio es el número de participantes en la muestra. Si bien es cierto que el número de participantes nos permite obtener una visión general de los comportamientos sexuales online de hombres y mujeres con diferentes orientaciones 
sexuales, sería necesario ampliar el muestreo para poder garantizar la generalizabilidad de los datos. Otra de las limitaciones tendría que ver con el procedimiento de reclutamiento. Puede ser que, aunque hayamos llevado a cabo diferentes estrategias de difusión, las personas que presenten un problema de adicción y hayan accedido al cuestionario por una búsqueda activa de información y/o tratamiento también estén sobrerrepresentadas en el estudio, lo que podría suponer que los resultados obtenidos no se ajusten exactamente a la realidad de la población general.

Con el propósito de subsanar estas limitaciones y avanzar en la investigación de este campo, consideramos necesario realizar estudios que evalúen todos estos aspectos de una forma mucho más comprehensiva en cada uno de los grupos analizados. Todavía no conocemos en profundidad las manifestaciones diferenciales del comportamiento sexual online en diferentes grupos de población (p.e., población mayor, población LGTBI, etc.), lo que todavía nos aleja del objetivo de conocer cuál es el impacto de internet en nuestra sexualidad y, sobre todo, de saber qué factores pueden favorecer la aparición de una adicción y cómo poder realizar una buena prevención e intervención en los casos que se requiera.

\section{Financiación}

Esta investigación se realizó gracias al apoyo económico de la Universitat Jaume I de Castellón (proyectos P1.1B2012-49, P1.1B2015-82 y UJI-B2018-42).

\section{Referencias}

Ballester-Arnal, R., Castro-Calvo, J., García-Barba, M., Ruiz-Palomino, E., y Gil-Llario, M. D. (2020). Prevalence, types, motives and problematic engagement in Online Sexual Activities (OSAs) across the lifespan: a largescale cross-sectional study. Manuscript Submitted for Publication.

Ballester-Arnal, R., Castro-Calvo, J., Gil-Llario, M. D., y Gil-Julià, B. (2016a). Cybersex Addiction: A Study on Spanish College Students. Journal of Sex \& Marital Therapy, 43(6), 567-584. https://doi.org/10.1080/009262 $3 \times .2016 .1208700$

Ballester-Arnal, R., Castro-Calvo, J., Gil-Llario, M. D., y Giménez-García, C. (2014). Relationship status as an influence on cybersex activity: cybersex, youth, and steady partner. Journal of Sex \& Marital Therapy, 40(5), 444-456. https://doi.org/10.1080/0092623X.2013.772549

Ballester-Arnal, R., Gil-Llario, M. D., Giménez-García, C., Castro-Calvo, J., y Cardenas-López, G. (2017). Sexuality in the internet era: expressions of Hispanic adolescent and young people. Sexual Addiction \& Compulsivity, 24(3), 140-155. https://doi.org/10.1080/10720162.2017.1329041

Ballester-Arnal, R., Gil-Llario, M. D., Gómez-Martínez, S., y Gil-Julià, B. (2010). Psychometric properties of an instrument for assessing cyber-sex addiction. Psicothema, 22(4), 1048-1053.

Ballester-Arnal, R., Giménez-García, C., Gil-Llario, M. D., y Castro-Calvo, J. (2016b). Cybersex in the "Net generation": Online sexual activities among Spanish adolescents. Computers in Human Behavior, 57, $261-266$. https://doi.org/10.1016/j.chb.2015.12.036

Böthe, B., Vaillancourt-Morel, M. P., Bergeron, S., y Demetrovics, Z. (2019). Problematic and non-problematic pornography use among LGBTQ adolescents: A systematic literature review. Current Addiction Reports, 6(4), 478-494. https://doi.org/10.1007/s40429-019-00289-5 
Carnes, P. J., Delmonico, D. L., y Griffin, E. (2001). In the Shadows of the Net: Breaking Free of Compulsive Online Sexual Behavior. Hazelden.

Castro-Calvo, J., Gil-Llario, M. D., Giménez-García, C., Gil-Juliá, B., y Ballester-Arnal, R. (2020). Ocurrence and clinical characteristics of Compulsive Sexual Behavior Disorder (CSBD): a cluster analysis in two independent community samples. Journal of Behavioral Addictions. https://doi.org/10.1556/2006.2020.00025

Castro-Calvo, J., Giménez-García, C., Gil-Llario, M. D., y Ballester-Arnal, R. (2018). Motives to engage in Online Sexual Activities and their links to an excessive and problematic use: a Systematic Review. Current Addiction Reports, 5(4), 491-510. https://doi.org/https://doi.org/10.1007/s40429-018-0230-y

Castro-Calvo, J., García-Barba, M., Gil-Juliá, B., Morell-Mengual, V., y Ballester-Arnal, R. (2018). Motivos para el consumo de cibersexo y su relación con el grado de severidad. Revista INFAD de Psicología. International Journal of Developmental and Educational Psychology., 7(1), 93-102.

Cooper, A. (1998). Sexually Compulsive Behavior. Contemporary Sexuality, 32(4), 1-3.

Cooper, A., y Griffin-Shelley, E. (2002). The internet:The next sexual revolution. In A. Cooper (Ed.), Sex \& the internet: A guidebook for clinicians (pp. 1-15). Brunner-Routledge.

Courtice, E. L., y Shaughnessy, K. (2018). The Partner Context of Sexual Minority Women's and Men's Cybersex Experiences: Implications for the Traditional Sexual Script. Sex Roles, 78(3-4), 272-285. https://doi. org/10.1007/s11199-017-0792-5

Daneback, K., Sevcikova, A., Månsson, S. A., y Ross, M. W. (2013). Outcomes of using the internet for sexual purposes: Fulfillment of sexual desires. Sexual Health, 10, 26-31. https://doi.org/10.1071/SH11023

Daneback, K., Cooper, A., y Mansson, S. (2005). An Internet study of cybersex participants. Archives of Sexual Behavior, 34, 321-328. https://doi.org/10.1007/s10508-005-3120-z

Delmonico, D. L. (1997). Cybersex: High tech sex addiction. Sexual Addiction \& Compulsivity: The Journal of Treatment and Prevention, 4(2), 159-167. https://doi.org/10.1080/10720169708400139

Duffy, A., Dawson, D. L., y das Nair, R. (2016). Pornography Addiction in Adults: A Systematic Review of Definitions and Reported Impact. The Journal of Sexual Medicine, 13(5), 760-777. https://doi.org/10.1016/j. jsxm.2016.03.002

Kafka, M. P. (2013). The Development and Evolution of the Criteria for a Newly Proposed Diagnosis for DSM-5: Hypersexual Disorder. Sexual Addiction \& Compulsivity, 20(1-2), 19-26. DOI: 10.1080/10720162.2013.768127

Kardefelt-Winther, D. (2014). A conceptual and methodological critique of internet addiction research: Towards a model of compensatory internet use. Computers in Human Behavior, 31(1), 351-354. https://doi. org/10.1016/j.chb.2013.10.059

Rochat, L., Bianchi-Demicheli, F., Aboujaoude, E., y Khazaal, Y. (2019). The psychology of "swiping": A cluster analysis of the mobile dating app Tinder. Journal of Behavioral Addictions, 8(4), 804-813. https://doi. org/10.1556/2006.8.2019.58

Ross, M. W., Tikkanen, R., y Mansson, S. (2000). Differences between Internet samples and conventional samples of men who have sex with men: implications for research and HIV interventions. Social Science \& Medicine, 51, 749-758. https://doi.org/10.1016/S0277-9536(99)00493-1

Seal, D. W., Benotsch, E. G., Green, M., Snipes, D. J., Bull, S. S., Cejka, A., Lance, S. P., y Nettles, C. D. (2015). The Use of Internet Chat Rooms to Meet Sexual Partners: A Comparison of Non-Heterosexually Identified Men with Heterosexually Identified Men and Women. International Journal of Sexual Health, 27(1), 1-15. https://doi.org/10.1080/19317611.2014.918921

Shaughnessy, K., Byers, E. S., y Walsh, L. (2011). Online Sexual Activity Experience of Heterosexual Students: Gender Similarities and Differences. Archives of Sexual Behavior, 40(2), 419-427. https://doi.org/10.1007/s10508010-9629-9

Smith, M. (2013). Youth Viewing Sexually Explicit Material Online: Addressing the Elephant on the Screen. Sexuality Research and Social Policy, 10(1), 62-75. https://doi.org/10.1007/s13178-012-0103-4

Træen, B., y Daneback, K. (2013). The use of pornography and sexual behaviour among Norwegian men and wom- 
en of differing sexual orientation. Sexologies, 22(2), e41-e48. https://doi.org/10.1016/j.sexol.2012.03.001

Wéry, A., y Billieux, J. (2016). Online sexual activities: An exploratory study of problematic and non-problematic usage patterns in a sample of men. Computers in Human Behavior, 56(October 2017), 257-266. https:// doi.org/10.1016/j.chb.2015.11.046

Wéry, A., y Billieux, J. (2017). Problematic cybersex: Conceptualization, assessment, and treatment. Addictive Behaviors, 64, 238-246. https://doi.org/10.1016/j.addbeh.2015.11.007

Wolak, J., Mitchell, K., y Finkelhor, D. (2007). Unwanted and wanted exposure to online pornography in a national sample of youth internet users. Pediatrics, 179(2), 247-257. https://doi.org/10.1542/peds.2006-1891 